\title{
DESIGN AND REAL-TIME MEASUREMENT OF HSDPA EQUALIZERS
}

\author{
Stefan Geirhofer, Christian Mehlführer, and Markus Rupp
}

\author{
Institute of Communications and Radio-Frequency Engineering \\ Vienna University of Technology \\ Gusshausstrasse 25/389, A-1040 Vienna, Austria
}

Email: \{sgeirhof, chmehl, mrupp $\} @$ nt.tuwien.ac.at

\begin{abstract}
The High Speed Downlink Packet Access (HSDPA) is an extension to UMTS that is tailored to high packet data throughput up to $14.4 \mathrm{Mbps}$. In order to reach these high data rates, it is indispensable for the receiver to cancel Multiple Access Interference (MAI) as to obtain a sufficiently high channel quality. In practice this renders the usage of conventional RAKE receivers impossible and makes equalization attractive to circumvent these problems.

This paper describes and compares known equalization schemes (MMSE, selected adaptive techniques) as well as new proposals (MMSE-RAKE). Other than related publications, the performance evaluation is based on measurements over a physical channel at $2.45 \mathrm{GHz}$ and is hence truly representative for a real HSDPA system. Further, the comparison is drawn both on performance and complexity, keeping a real-time implementation in focus.
\end{abstract}

\section{INTRODUCTION}

HSDPA uses W-CDMA for multiuser communication and thus orthogonal spreading codes are used to separate different users in the downlink. However, the orthogonality of these codes is destroyed by the multipath characteristics of the channel, resulting in MAI. Whereas schemes that do not take this into account (e.g. the conventional RAKE receiver) have a considerably poor performance, introducing an equalizer significantly improves the bit error rate by (partly) restoring orthogonality. Reducing the bit error rate in turn ameliorates the overall performance of HSDPA because high data rates will be allocated more often. Additionally, the number of retransmissions is reduced in this way.

After briefly introducing the signal model, the conventional RAKE receiver is presented in Sec. 2 and compared to an MMSE equalizer as well as to selected adaptive methods. We consider pilot-assisted schemes as well as a blind technique employing a constant modulus argument. In Sec. 3 the measurement configuration is presented followed by the measurement results in Sec. 4, the emphasis of this work. A conclusion summarizes this paper's contributions.

Signal Model: The signal model is depicted in Fig. 1. We consider a number of bit streams $b_{n}[i]$ as input. In particular, $b_{1}, \ldots, b_{N-1}$ denote the user's data sequences and $b_{0}$ is the pilot,

This work was funded by the Christian Doppler Laboratory for Design Methodology of Signal Processing Algorithms as well as Kplus, Infineon Technologies and the ARC Seibersdorf Research $\mathrm{GmbH}$ (ARCS) through ftw. Projects C3 and C9.

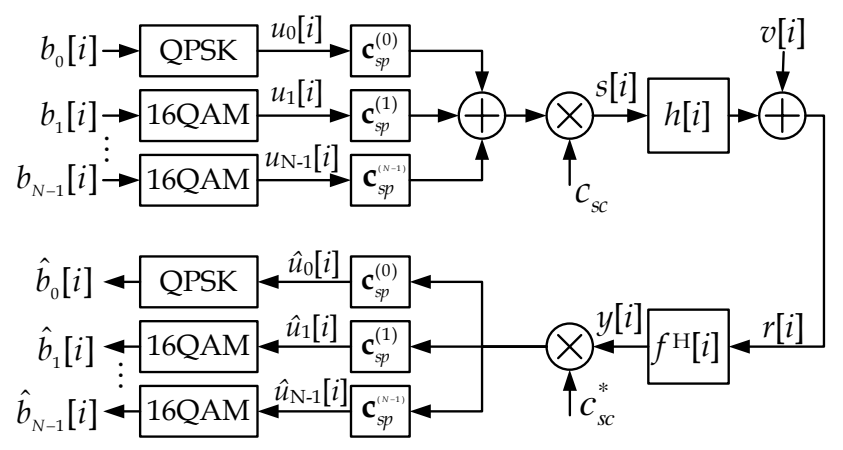

Fig. 1. Signal model

a deterministic sequence that can be used for channel estimation or pilot-assisted adaptation. The pilot is mapped with QPSK, all user channels are modulated with 16QAM, i.e. the constellation that is used in HSDPA for high data rates. The signals are spread with Walsh-Hadamard sequences $\mathbf{c}_{\mathrm{sp}}$ of length $N=16$ (the pilot is spread with a factor of 256) and scrambled with $c_{\mathrm{sc}}[i]$. Subsequently, the signals pass the unknown channel $h[i]$ (composed of a channel emulator and the RF-frontend). Additive white Gaussian noise $v[i]$ is added, forming the received signal $r[i]$. This signal is equalized with $f[i]$ to get $y[i]$ and finally descrambled, despread and demodulated to obtain estimates for the transmitted bits. In the following $L_{h}$ will denote the length of the channel in chips and $L_{f}$ stands for the length of the equalizer in taps.

\section{MATHEMATICAL ANALYSIS}

In this section we first present the design of the conventional RAKE receiver and compare it to the MMSE equalizer. Subsequently, selected adaptive techniques promising a good performance are discussed.

\subsection{RAKE Receiver}

The conventional RAKE receiver approximately implements a matched filter for the channel impulse response. Thus, it has the structure of a tapped delay line as depicted in Fig. 2. The signal $r[i]$ is first delayed and then descrambled, despread and combined according to Maximum Ratio Combining (MRC). The coefficients $a_{l}$ are found through channel estimation. 


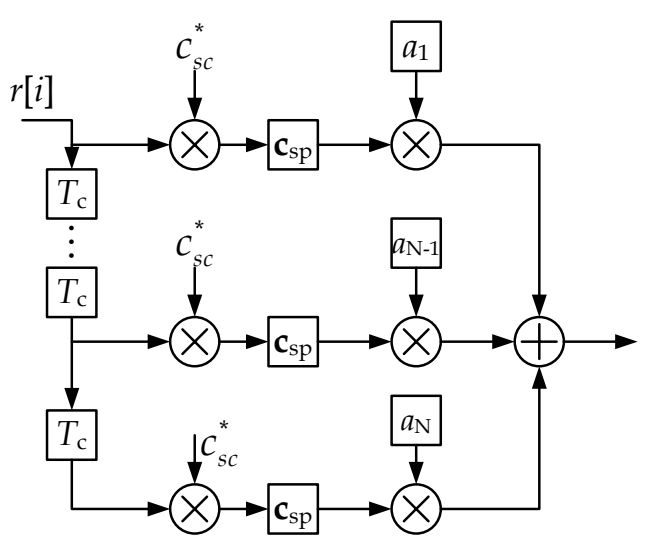

Fig. 2. RAKE receiver

\subsection{MMSE Equalizer}

The goal of an MMSE equalizer is to find the equalizer's coefficient vector $\mathbf{f}$ such that the equalized receive signal $y[i]$ is as close as possible to the transmitted signal $s[i]$. The optimization criterion formulates this in the Minimum Mean Squared Error (MMSE) sense

$$
\mathbf{f}=\arg \min _{\mathbf{f}} J_{1}(\mathbf{f})=\arg \min _{\mathbf{f}} \mathbb{E}\left\{\left|\mathbf{f}^{H} \mathbf{r}_{i}-s_{i-\tau}\right|^{2}\right\},
$$

where $s_{i-\tau}$ is a delayed version of the transmitted signal at the chip-rate,

$$
\mathbf{r}_{i}=\left[r[i], r[i-1], \ldots, r\left[i-L_{f}+1\right]\right]^{T}
$$

is a vector of the $L_{f}$ last received samples and $\tau$ is the inevitable delay that is caused by the system (filters and channel).

A solution to (1) is given in [1]

$$
\mathbf{f}=\sigma_{s}^{2}\left(\sigma_{s}^{2} \mathbf{H} \mathbf{H}^{H}+\sigma_{v}^{2} \mathbf{I}\right)^{-1} \mathbf{H} \mathbf{e}_{\tau}
$$

In this formula the $L_{f} \times L_{h}+L_{f}-1$ sized matrix

$$
\mathbf{H}=\left[\begin{array}{ccccc}
h_{0} & \cdots & h_{L_{h}-1} & & 0 \\
& \ddots & \ddots & \ddots & \\
0 & & h_{0} & \cdots & h_{L_{h}-1}
\end{array}\right]
$$

denotes the convolution with the channel's impulse response, $\mathbf{I}$ is the identity matrix and $\mathbf{e}_{\tau}$ is a unit vector with a one at the $\tau$-th position. The matrix $\mathbf{H}$ is found by estimating the channel.

Complexity: Calculating the equalizer's coefficients involves computing a matrix inverse of size $L_{f} \times L_{f}$. Although there are efficient implementations that, for instance, exploit the Toeplitz structure [2] this remains computationally expensive. Whether MMSE equalization is feasible for a real-time implementation also depends on how fast the channel varies, i.e. how frequent the coefficients have to be computed.

\subsection{MMSE-RAKE Receiver}

The conventional RAKE receiver as outlined above is optimal for an AWGN channel, however this is never the case in a practical HSDPA setup. The interference that is generated through other

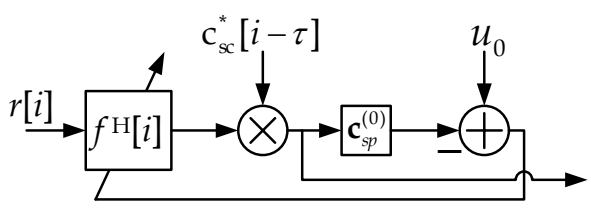

(a) Symbol-rate adaptive equalizer

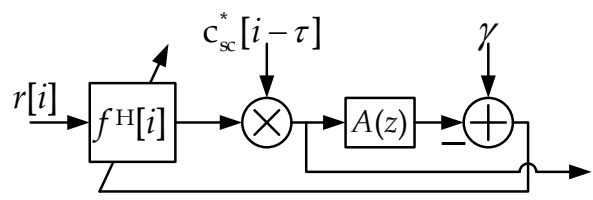

(b) Chip-rate adaptive equalizer

Fig. 3. Adaptive equalization methods

user channels (or even one's own channel) is not reduced at all and severely limits the performance.

We can improve the RAKE receiver by combining the paths with respect to a more effective criterion. Similar to the MMSE receiver, we can impose the cost-function

$$
J_{2}(\mathbf{a})=\mathbb{E}\left\{\left|\mathbf{a}^{H} \tilde{\mathbf{r}}_{i}-s_{i-\tau}\right|^{2}\right\},
$$

where $\mathbf{a}^{H}$ denotes the combining coefficients and $\tilde{\mathbf{r}}_{i}$ is a corresponding vector of the received samples (determined by the finger placement). The number of fingers is $L_{a}<L_{h}$.

The solution is similar to (3), however the definition of $\mathbf{H}$ is now different since it has to reflect the finger placement. The Toeplitz structure is lost, but the size of the matrix decreases from $L_{f}$ to $L_{a}$, i.e. reducing the computational cost.

\subsection{Symbol-Rate Adaptive Equalization}

By using adaptive equalization, it is possible to reduce the computational complexity drastically. Let's first consider the equalizer depicted in Fig. 3(a). The incoming signal is first equalized with f and then descrambled and despread with the pilot's code (shortened to length $N=16$ ) to get an estimate for the pilot symbols. Since this sequence is known, we can use the estimate to update the equalizer's coefficients. Mathematically, this can be expressed as

$$
\hat{u}_{0}[i]=\tilde{\mathbf{c}}_{i N}^{H}\left[\begin{array}{llll}
\mathbf{f}^{H} & & & \mathbf{0} \\
& \mathbf{f}^{H} & & \\
& & \ddots & \\
& & & \mathbf{f}^{H}
\end{array}\right] \mathbf{r}_{i N}
$$

where the descrambling and despreading operation have been merged into the time-variant code vector $\tilde{\mathbf{c}}$, and with

$$
\mathbf{r}_{i N}=\left[r[i N], r[i N-1], \ldots, r\left[i N-N-L_{f}+2\right]\right]^{T} .
$$

We can rewrite this as

$$
\hat{u}_{0}[i]=\mathbf{f}^{H}\left[\begin{array}{cccc}
\tilde{\mathbf{c}}_{i N}^{H} & & & \mathbf{0} \\
& \tilde{\mathbf{c}}_{i N}^{H} & & \\
& & \ddots & \\
& & & \tilde{\mathbf{c}}_{i N}^{H}
\end{array}\right] \mathbf{r}_{i N}=\mathbf{f}^{H} \tilde{\mathbf{C}}_{i N} \mathbf{r}_{i N} .
$$


We formulate the cost function such that the error associated with estimating the pilot symbol is minimum, i.e.

$$
J_{3}(\mathbf{f})=\mathbb{E}\left\{\left|u_{0}[i]-\hat{u}_{0}[i]\right|^{2}\right\} .
$$

The above cost function is minimized using steepest-descent

$$
\mathbf{f}^{(i+1)}=\mathbf{f}^{(i)}-\mu \nabla J_{3}\left(\mathbf{f}^{(i)}\right) .
$$

The gradient in (10) is found to be

$$
\frac{\partial J_{3}}{\partial \mathbf{f}^{*}}=-\mathbb{E}\left\{\left(u_{0}[i]-\hat{u}_{0}[i]\right)^{*} \tilde{\mathbf{C}}_{i N} \mathbf{r}_{i N}\right\} .
$$

\subsection{Chip-Rate Adaptive Equalization}

The pilot-assisted equalizer described above updates its coefficients only every $N$ chips (due to the despreading operation). For this reason [1] proposes to replace the despreading operation with a low-pass filter. This is indeed possible because the pilot sequence is constant and the undesired interference is zero-mean. The mathematical background of this algorithm is explained in detail in [1].

An important design consideration of this scheme is how to choose the low-pass filter in Fig. 3(b). Throughout this paper we shall assume the first order filter

$$
A(z)=\frac{1-\rho}{1-\rho z^{-1}} .
$$

Thus we arrive at two design parameters that influence the performance: the step-size $\mu$ and the parameter $\rho$. Ultimately, $\rho$ should be chosen large enough to sufficiently suppress MAI, but small enough to track channel variations (which cannot be distinguished from MAI).

\subsection{Constant Modulus Algorithm}

The Constant Modulus Algorithm (CMA) makes use of the fact that the pilot channel is mapped with QPSK, a constellation with constant modulus. CMA is 'blind' and hence requires no other information, i.e. it does not use the fact that the pilot symbols are known. We formulate the cost function according to [3] ( $\gamma$ is the constant modulus)

$$
J_{4}(\mathbf{f})=\mathbb{E}\left\{\left(\left|\hat{u}_{0}\right|^{2}-\gamma^{2}\right)^{2}\right\} .
$$

We again use the steepest-descent approach of $(10)$. The gradient can be found as

$$
\frac{\partial J_{4}}{\partial \mathbf{f}^{*}}=-2 \mathbb{E}\left\{\left(\left|\hat{u}_{0}\right|^{2}-\gamma^{2}\right) \hat{u}_{0}^{*}[i] \tilde{\mathbf{C}}_{i N} \mathbf{r}_{i N}\right\} .
$$

It should be noted that it is not possible to use multiuser CMA as described in [3] for HSDPA, since the user channels are mapped with 16QAM. A good initial guess is important for this algorithm since it significantly speeds up the convergence time (the gradient is very small close to an initial value of all-zero).

\section{MEASUREMENT SETUP}

In contrast to other publications this paper compares the equalization schemes based on measurements over a physical channel. The MIMO testbed developed at the Institute of Communications and Radio-Frequency Engineering [4, 5] has been used for this purpose. The measurement setup is depicted in Fig. 4.

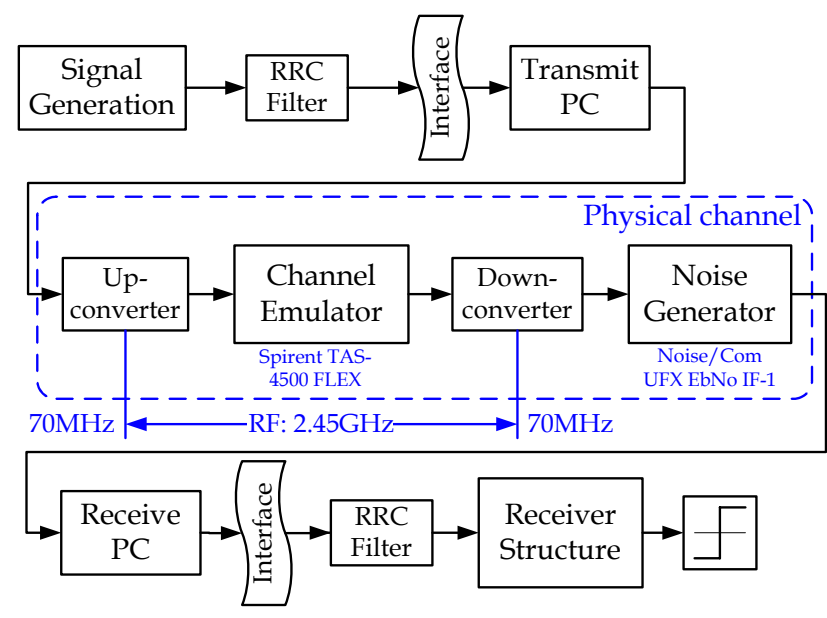

Fig. 4. Measurement setup

Signal generation: At first, the HSDPA channels are generated at the chip-rate according to the 3GPP specification. However, in order to arrive at a physical signal we need to bandlimit them to approximately $5 \mathrm{MHz}$ with a root raised cosine (RRC) filter (rolloff factor 0.22) as specified in [6].

Physical channel: In the next step, we interface the transmit PC of the testbed, which in turn generates physical signals at $70 \mathrm{MHz}$. These are then upconverted to $2.45 \mathrm{GHz}$ and passed through a Spirent TAS-4500 FLEX channel emulator configured for the ITU Pedestrian B channel model [6]. Subsequently, the signal is downconverted again and noise is added with the Noise/Com UFXEbNo. Finally, the resulting signal is detected by the receive PC and passed on to a computer cluster for further processing.

Receive Processing: At first slot synchronization is accomplished by correlating the received samples (fractionally chip-spaced) with the known synchronization preamble. Then, the signals are filtered with a chip-matched RRC filter and processed with the different receivers.

Measurement Verification: The setup has been extensively tested to ensure correct operation and accuracy. For this purpose the channel emulator was configured for an AWGN and a flat Rayleigh fading channel, respectively. The results showed an almost perfect fit with the analytical formulas. The largest abberation occurred at $E_{b} / N_{0}=13 \mathrm{~dB}$ and amounted to $0.15 \mathrm{~dB}$.

\section{MEASUREMENT RESULTS}

This section analyzes the measurement results and compares the selected equalization schemes. We show how to choose design parameters as to find a compromise between performance and complexity and specifically address pitfalls that might hinder a realtime implementation.

In all scenarios (except the tracking behavior) a static Pedestrian B channel has been used. Further, the power allocation devoted $80 \%$ of the power to the user channels and $20 \%$ to the pilot channel. The synchronization channel was only transmitted as a preamble (for the testbed to get synchronized; no contribution to the power allocation), but not jointly with the other channels. In a practical system, the interference of the synchronization channel can be canceled because the sequence is known at the receiver [7]. 


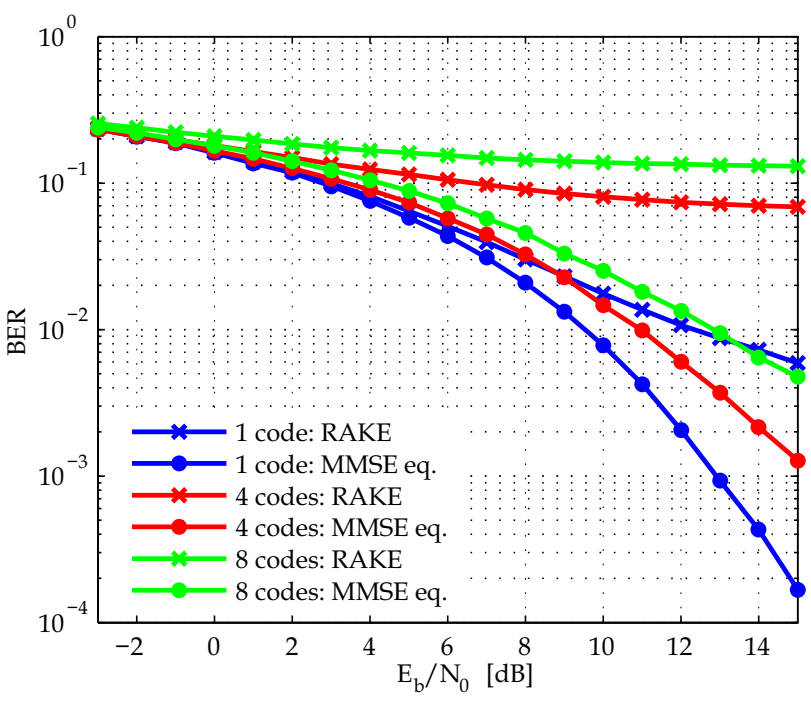

Fig. 5. Comparison of the RAKE receiver and the MMSE equalizer (MMSE: $L_{f}=48$, RAKE: $L_{a}=16$, static Ped. B).

\subsection{MMSE Equalizer vs. RAKE Receiver}

The performance of the MMSE equalizer is compared to the RAKE receiver in Fig. 5 for different numbers of active CDMA codes. The measurements show that a significant performance gain is associated with the equalizer and that the overall system is no longer interference limited.

The bit error rate performance decreases as the number of active codes is increased, since more interference is generated with respect to each individual channel (the power for all channels is kept constant).

In addition, we also measured the performance of fractionally chip-spaced MMSE equalizers. Although the higher sampling rate results in additional complexity (larger matrix-inverse) the results were only marginally better compared to the chip-spaced solution.

\subsection{Optimal Equalizer Length}

An important design consideration for implementing the equalizer is to choose the length $L_{f}$ adequately. In fact, choosing $L_{f}$ large (several multiples of the channel delay $L_{h}$ ) improves the accuracy, but at the cost of inverting a large matrix of size $L_{f} \times L_{f}$. Thus, it is necessary to find a suitable tradeoff, which naturally depends on the channel's characteristics.

In Fig. 6 the BER for the MMSE equalizer is shown over $L_{f}$ for selected values of $E_{b} / N_{0}$. For the measurements the Pedestrian B channel was used, which has a maximum delay of $L_{h}=16$ chips. The best performance is achieved if $L_{f}$ is maximum ( $L_{f}=3 L_{h}=48$ chips). As $L_{f}$ is decreased the BER stays about the same until we reach $L_{f}=32$. Decreasing $L_{f}$ below this value leads to a monotonous degradation in BER of almost one decade. From the implementation perspective choosing $25<L_{f}<32$ is attractive because we save computational cost (matrix inverse) while retaining good performance.

\subsection{Adaptive Equalizers}

In Fig. 7 the performance of the selected equalizer structures is shown and quantitatively compared to the MMSE method in Tab. 1

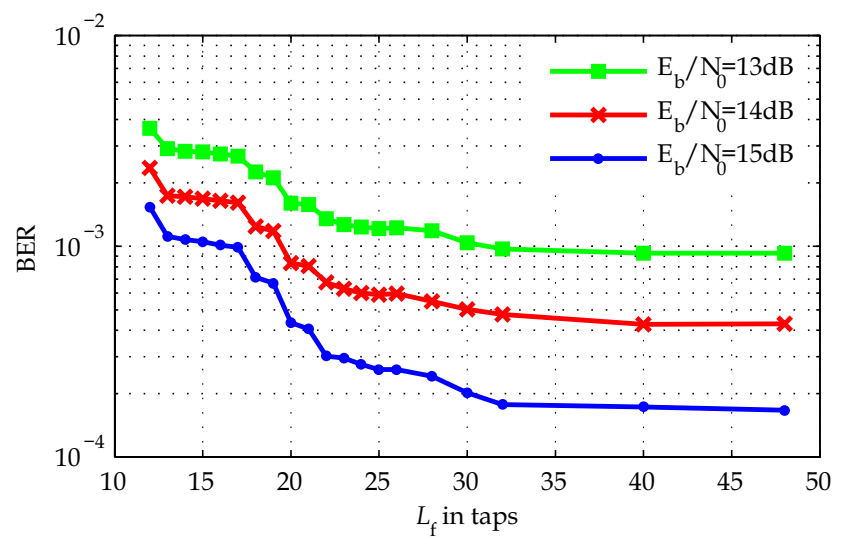

Fig. 6. BER for the MMSE equalizer as a function of $L_{f}$ for selected values for $E_{b} / N_{0}$ (static Ped. B).

and Tab. 2. For $E_{b} / N_{0}>10 \mathrm{~dB}$ all adaptive schemes show a performance similar to the MMSE method. The convergence behavior for the adaptive methods is analyzed in Tab. 3. In Fig. 6 and Fig. 7 only one user code is active, corresponding to the first case in Fig. 5 .

Symbol-rate adaptive equalizer: The symbol-rate adaptive equalizer almost lives up to the performance of the MMSE equalizer. The largest abberation occurs at $14 \mathrm{~dB}$ and amounts to $0.5 \mathrm{~dB}$. Convergence is reached after approximately 5 slots, the MSE improvement of the adaptive scheme is $\approx 35 \mathrm{~dB}$ (see Tab. 3).

Chip-rate adaptive equalizer: The performance of the chip-rate adaptive equalizer is almost identical to the MMSE method up to $E_{b} / N_{0}=10 \mathrm{~dB}$, then the performance saturates. Convergence is reached after 2 slots, the MSE improvement is approximately $25 \mathrm{~dB}$. These values can be adjusted with $\rho$, trading off convergence speed with accuracy ( $\rho=0.95$ for the above example).

CMA-equalizer: The CMA equalizer shows a poor performance for $E_{b} / N_{0}<10 \mathrm{~dB}$. However, at high $E_{b} / N_{0}$ the performance becomes very good outperforming the other schemes at $E_{b} / N_{0}=15 \mathrm{~dB}$. CMA converges slowly (12 slots) and shows an MSE improvement of $31 \mathrm{~dB}$.

MMSE-RAKE: The MMSE-RAKE outperforms the conventional RAKE by about $2-3 \mathrm{~dB}$ at $12<E_{b} / N_{0}<15$. In Fig. $7 L_{a}=10$ fingers were used.

\subsection{Tracking Behavior}

In addition to comparing the convergence speed for the selected adaptive equalizers, we also analyzed the tracking behavior in time-variant channels. Using the channel emulator a flat (1-tap) Rayleigh fading channel for a Doppler velocity of $v=30 \mathrm{~km} / \mathrm{h}$ was configured. The results are shown in Fig. 8 for a time period of five consecutive HSDPA frames and compared to the 'ideal' case gained through channel estimation. Indeed, all of the adaptive techniques were able to track the time-variant channel. Although the chip-rate adaptive equalizer updates its coefficients $N$ times faster than the other schemes the tracking performance is slightly worse. This is due to the fact that the updates at the symbol-rate are still fast enough to accurately track the channel. Therefore the chip-rate algorithm's superiority in this respect diminishes. It also has to be noted that this method has only been tested for a first-order low-pass filter. Deploying a higher order filter might improve the performance. 


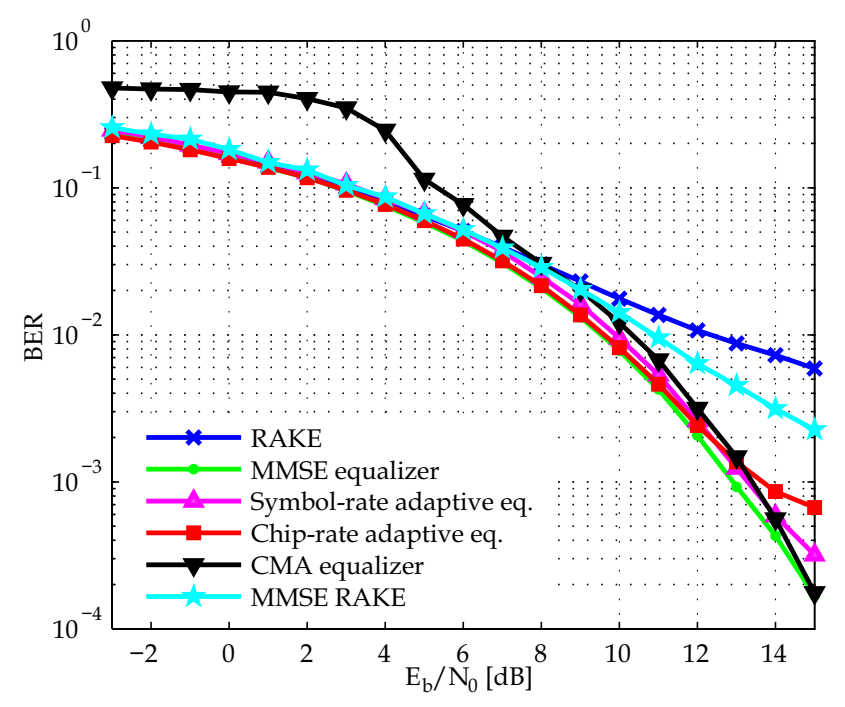

Fig. 7. BER performance compared to the MMSE solution for the presented equalizers (static Ped. B; see also Tab. 1).

\section{CONCLUSION}

In conclusion we have proven by measurement that equalization clearly outperforms the conventional RAKE receiver. The MMSE equalizer shows a performance gain of more than one decade in BER for representative conditions. Additionally, we analyzed how to choose the equalizer length for this scheme.

Moreover, we presented results for various adaptive techniques that are computationally efficient. Their performance is very close to the MMSE method and further allows for tracking time-variant channels. Since the adaptive methods were proven to converge towards the MMSE solution, the convergence time can drastically be reduced by combining the two methods, i.e. computing a good initial guess with the MMSE method.

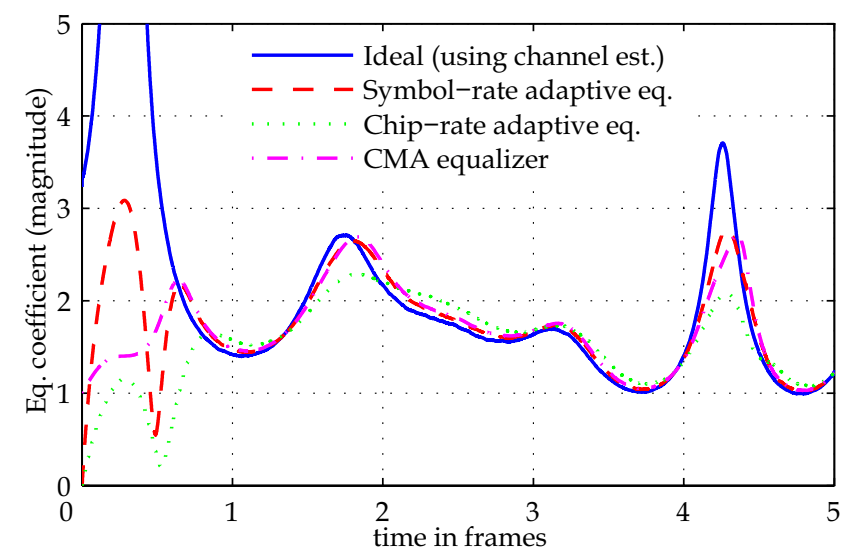

Fig. 8. Tracking behavior of the adaptive schemes for a flat (1tap) Rayleigh fading channel with Doppler velocity $v=30 \mathrm{~km} / \mathrm{h}$. Comparison with ideal results gained through channel estimation.
Table 1. Quantitative comparison of the equalization schemes shown in Fig. 7. BER at given $E_{b} / N_{0}$.

\begin{tabular}{|c|c|c|c|c|c|}
\hline$E_{b} / N_{0} / \mathrm{dB}$ & 6 & 8 & 10 & 12 & 14 \\
\hline MMSE & 0.043 & 0.021 & 0.0078 & $2.1 \cdot 10^{-}$ & $4 \cdot 10^{-4}$ \\
\hline RAKE & 0.051 & 0.030 & 0.018 & 0.011 & $7.3 \cdot 10^{-3}$ \\
\hline Symb.-rate eq. & 0.051 & 0.025 & 0.0095 & $2.6 \cdot 10$ & $3 \cdot 10^{-4}$ \\
\hline Chip-rate eq. & 0.045 & 0.022 & 0.0082 & $2.4 \cdot 10^{-3}$ & $7 \cdot 10^{-4}$ \\
\hline CMA & 0.077 & 0.047 & 0.012 & $3.1 \cdot 10$ & $2 \cdot 10^{-4}$ \\
\hline$M M S E-R A K E$ & 80.052 & 0.029 & 0.014 & $6.4 \cdot 10^{-3}$ & $2.3 \cdot 10^{-3}$ \\
\hline
\end{tabular}

Table 2. Quantitative comparison of the equalization schemes shown in Fig. 7. Loss/dB compared to MMSE equalizer for specified BER.

\begin{tabular}{|c|c|c|c|c|c|}
\hline BER & 0.1 & 0.01 & $5 \cdot 10^{-3}$ & $1 \cdot 10^{-3}$ & $5 \cdot 10^{-4}$ \\
\hline RAKE & 0.21 & 2.82 & $>4$ & - & - \\
\hline Symb.-rate eq. & 0.50 & 0.38 & 0.36 & 0.38 & 0.46 \\
\hline Chip-rate eq. & 0.03 & 0.08 & 0.13 & 0.76 & - \\
\hline$C M A$ & 2.57 & 0.80 & 0.66 & 0.50 & 0.29 \\
\hline$M M S E-R A K E$ & 0.44 & 1.36 & 1.97 & $>2$ & - \\
\hline
\end{tabular}

Table 3. Convergence behavior for the presented adaptive techniques. Definitions: convergence time: $|e[i]|^{2}$ reaches $1 \%$ of $|e[0]|^{2}$; MSE improvement: $|e[0]|^{2} /|e[\infty]|^{2} ; 1$ frame $=15$ slots.

\begin{tabular}{ccc}
\hline & Convergence time/slots & Improvement/dB \\
\hline \hline Symb.-rate eq. & 4.6 & 35.2 \\
\hline Chip-rate eq. & 2.4 & 25.2 \\
\hline CMA & 12.2 & 31.0 \\
\hline
\end{tabular}

\section{REFERENCES}

[1] P. Schniter and A. R. Margetts, "Adaptive Chip-Rate Equalization of Downlink Multirate Wideband CDMA," Proc. 36th Asilomar Conf. on Signals, Systems and Computers, vol. 2, pp. 1228-1232, Nov. 2002.

[2] L. Mailaender, "Low-complexity implementation of CDMA downlink equalization," Second International Conf. on $3 G$ Mobile Communication Technologies, no. 477, pp. 396-400, Mar. 2001.

[3] S. Weiss, M. Hadef, and M. Rupp, "Blind chip-rate equalisation for DS-CDMA downlink receiver," 37th Asilomar Conf. on Signals, Systems, and Computers, vol. 2, pp. 1283-1287, Nov. 2003.

[4] S. Caban, C. Mehlführer, R. Langwieser, A. L. Scholtz, and M. Rupp, "Real-Time Matlab Extension for MIMO-Testbeds," submitted to JASP special issue on MIMO testbeds, 2005.

[5] C. Mehlführer, S. Geirhofer, S. Caban, and M. Rupp, "A flexible MIMO testbed with remote access," EUSIPCO, 2005.

[6] 3rd Generation Partnership Project, "User Equipment (UE) radio transmission and reception (FDD)," Mar. 2004, 3GPP, TS 25.101 V6.4.0.

[7] M. Harteneck, M. Boloorian, S. Georgoulis, and R. Tanner, "Practical aspects of an HSDPA 14 Mbps terminal," 38th Asilomar Conf. on Signals, Systems and Computers, Nov. 2004. 